\title{
Agent Affecting Blood or Body Fluid
}

National Cancer Institute

\section{Source}

National Cancer Institute. Agent Affecting Blood or Body Fluid. NCI Thesaurus. Code C78275.

An agent that exerts an effect on the synthesis or function of blood, blood components or body fluids. 